\title{
Analysis of aerosol production and aerosol dispersion during otologic surgery
}

\author{
Manuel Gomez Serrano ${ }^{1}$ (1) $\cdot$ Andres Santiago-Saez ${ }^{2} \cdot$ Rosa Moreno Rodriguez $^{3} \cdot$ Andrea Lopez Salcedo $^{1} \cdot$ \\ Adriana Poch Perez-Botija ${ }^{1}$. William Aragones Sanzen-Baker ${ }^{1}$. Angel F. García Martín ${ }^{4}$. \\ Juan Antonio García García ${ }^{5}$ Maria Cruz Iglesias Moreno ${ }^{1}$
}

Received: 10 May 2021 / Accepted: 23 August 2021 / Published online: 3 September 2021

(c) The Author(s), under exclusive licence to Springer-Verlag GmbH Germany, part of Springer Nature 2021

\begin{abstract}
Background As a respiratory disease, the transmission of Coronavirus disease (COVID-19) is mainly caused by small droplets and aerosols. Healthcare personnel are particularly exposed during otologic surgery given the continuity with the nasopharynx, where the viral load is high, and the use of high-speed instruments. The purpose of the present study is to test a model of droplet dispersion produced in the performance of a drilling procedure on human bone to provide information about its distribution and size of the deposit in similar conditions to those of an operating theatre, to design different preventive measures.

Material and method A mastoidectomy and trans-labyrinthine approach were performed on an embalmed human corpse using for irrigation during drilling methylene blue dye in physiological saline solution (pss) at a concentration of $0.324 \mathrm{mg} /$ $\mathrm{mL}$. The distribution of the drops was stablished using semi-absorbent papers of size $52 \mathrm{~cm} \times 42 \mathrm{~cm}$ covering the area around the dissection field to a radius of $150 \mathrm{~cm}$ and on the corpse at different heights to check vertical dispersion. The collected deposit material was analysed with the microscope at different magnification objectives.

Results Droplets between $2 \mu \mathrm{m}$ and $2.6 \mathrm{~cm}$ were obtained. The visualization of the coloured droplets in the horizontal plane at a magnification of 1.5 was detected at $150 \mathrm{~cm}$ from the focus of emission of milling particles.

Discussion According to our study, bone drilling with high speed motors under continuous saline irrigation in a haemorrhagic surgical field increases the amount of aerosols exposing healthcare personnel to additional airbone particles. This risk does not end in the operating rooms as particles smaller than $2 \mu \mathrm{m}$ can be suspended in the air for hours and could exit the operating theatre due to the use of positive pressure systems. Thus, the use of N95, FFP2, FFP3 or PAPRS should be considered and the development of hood systems to prevent the dispersion of aerosols during these procedures should be considered.
\end{abstract}

Keywords COVID-19 · Aerosol · Otologic surgery

\section{Introduction}

Coronavirus disease 2019 (COVID-19) was first documented in Wuhan, Hubei Province, China, in December 2019. Since then, it has spread exponentially worldwide with great health and economic impact [1]. Several transmission models of the different infectious agents are described in the literature such as human-to-human transmission, airborne, and other forms such as through a vector. For respiratory viruses, transmission via small droplets and aerosols is a fundamental route,

Manuel Gomez Serrano

manuelgomezserrano@gmail.com

Extended author information available on the last page of the article although its significance is not clearly established [2,3]. In general, infectious patients spread the virus through small viral particles enclosed within small encapsulated globules of mucus, saliva or water that they release when they speak, breathe, cough or sneeze. The behaviour of these particles will depend on their size, their weight, and different characteristics of the environment in which they are released such as air humidity or air turbulence that allows them to float for more or less time [4].

An aerosol is defined as the suspension of particles in a gas long enough to be transported for a few seconds. These particles can be solid, liquid or a combination of both. Commonly, in medical terminology, the word aerosol is accepted to refer only to particles that are less than or equal to $5 \mu \mathrm{m}$. 
On the other hand, these particles can also be classified according to where they are deposited in the respiratory tract. Thus, respirable aerosols are particles smaller than $5 \mu \mathrm{m}$ or even smaller than $2.5 \mu \mathrm{m}$ that are deposited along the respiratory bronchioles and alveoli. Thoracic aerosols are those particles of between 10 and $15 \mu \mathrm{m}$ that can reach the trachea and the bronchi. Finally, inhalable aerosols refer to particles of up to 100 or $200 \mu \mathrm{m}$ capable of penetrating through the nose by inhalation [5]. For a virus capable of infecting cells located throughout the respiratory tract, all these types of particles are important, especially if we take into account that they may also produce contagion through the conjunctival mucosa [6].

Otolaryngology specialists are particularly exposed to airborne diseases, particularly to coronavirus given the known high viral load in the upper airway of infected patients and the performance of multiple aerosol-generating procedures [7]. This occurs to such extent that, during the first wave of the pandemic, the American Academy of Otolaryngology, Head and Neck Surgery (AAO-HNS) recommended that all otolaryngologic processes, and especially those involving the upper airway, be deferred unless deemed medically necessary or until preoperative COVID-19 testing could be reliably performed. Additionally, it has been proven that, directly or indirectly, multiple viruses can often affect the ear from the pharynx or systemically, with the presence of these viruses being found by PCR of samples taken from these locations [1]. Unfortunately, there is limited data on middle ear and mastoid viral load. To understand the potential risk of otologic procedures in the context of an airborne viral pandemic, we must take into account both the viral load present in the middle ear and aerosolization during otologic procedures, especially if high-speed instruments, drills, are being used. Most otologic procedures can be classified as elective and should be postponed until the above conditions are met, however, there are also urgent and emergent procedures. Acute mastoiditis with bone destruction, complicated mastoiditis or complicated acute otitis media may require myringotomy and insertion of a ventilation tube and/or cortical mastoidectomy. Other urgent cases include intracranial tumours with brainstem compression, some cases of acute facial palsy, advanced cholesteatoma, cerebrospinal fluid fistulas, temporal bone neoplasms, post meningitic cochlear implants, and removal of infected cochlear implants.

Current anaesthesiology guidelines recommend orotracheal intubation avoiding ventilation with laryngeal masks, which provide less airway sealing and higher risk of aerosolization. During sinus and upper airway surgery, especially if electrical instruments are used, aerosolization of COVID19 virus can be extremely high. During otologic procedures virus aerosolization can also occur given the continuity with the nasopharynx, thus creating an open system with the environment and the nasopharynx, providing another possible route for aerosol generation despite orotracheal intubation. In addition, procedures on the mastoid bone present an increased risk of aerosol generation and exposure given the standard use of high-speed drills. There is a lack of data on the risk of viral exposure with the use of powered instruments during ear and lateral skull base surgery. Importantly, surgical masks have been tested to be ineffective in preventing inhalation of bone dust particles and N95 masks (filtering at least $95 \%$ of airborne particles) significantly decrease particulate exposure in this environment, but do not fully supress it [8]. Finally, the generated bone dust micro-spicules can penetrate the cornea in animal models and has been reported to serve as a potential vector for viral transmission and transconjunctival spread of COVID-19 [6].

The degree of particulate aerosolization generated during middle ear and cerebellopontine angle surgery with highspeed drills and its diffusion within the surgical suite may be such as to force the use of a power air-purifying respirator (PAPR) or, alternatively, N95 or FFP3 masks together with eye protection in the form of a full-face mask or sealed goggles for all personnel in the room.

The purpose of the present study is to test a model of droplet dispersion produced in the performance of a drilling procedure on human bone. This may provide information about its distribution, and size of the deposit in similar conditions similar to those of an operating theatre, to design different preventive measures of contagion in otologic emergency surgeries on COVID patients as mentioned above.

\section{Materials and methods}

We present a prospective observational study performed during May 2020, in a dissection room reproducing the conditions of an operating room, at a temperature of $24^{\circ} \mathrm{C}$.

A mastoidectomy and trans-labyrinthine approach were performed on a corpse using the usual surgical material.

Around a central table, a barrier of $200 \mathrm{~cm} \times 76 \mathrm{~cm}$ nonabsorbent white soakers was placed as a reference, coinciding with the head of an embalmed human corpse.

Semi-absorbent papers of size $52 \mathrm{~cm} \times 42 \mathrm{~cm}$ were used.

The equipment used for milling was an ANSPACH EG1 with a maximum speed of $80,000 \mathrm{rpm}$.

For irrigation during drilling, we used methylene blue dye (certistain) at a concentration of $12.5 \mathrm{~g}$ diluted in $1 \mathrm{~L}$ of physiological saline solution (pss). From this solution, $80 \mathrm{~mL}$ were taken and placed in a 3-L bag of pss, yielding a concentration of $0.324 \mathrm{mg} / \mathrm{mL}$. A valved serum delivery system connected to the bag with the dye dilution was used for irrigation while drilling with diamond, rough diamond and flutted spherical drills with diameters of 6, 4, 3, 2 and $1 \mathrm{~mm}$.

Three Canon Mark III cameras and an Iphone 11 titlting support system for zenithal recording were used. 
A hemispherical tent with a radius of $150 \mathrm{~cm}$ was mounted to protect the environment (OUTSUNNY ${ }^{\circledR}$ 84C-110).

\section{Method}

The head was placed on a central table in a supine surgical position with a $45^{\circ}$ contralateral cephalic partial rotation to reproduce an ear surgery (open mastoidectomy) and a lateral skull base approach (trans-labyrinthine approach of the pontocerebellar angle).

A perimetral barrier of soakers was established and the radius was modified by taking as a reference point the external auditory canal of the dissected ear. Soakers were positioned at a distance of $2 \mathrm{~m}$, approaching progressively every $15 \mathrm{~cm}$.

Irrigation during drilling was performed with the dyed saline solution mentioned above to allow better visualization of the volatile particles obtained during drilling.

After verifying the maximum distance at which the perimeter barrier was impacted with a $1.5 \times$ magnifying glass, we proceeded to establish this radius as a reference for our working model. A hemispherical tent of that radius $(150 \mathrm{~cm})$ was installed to reproduce the space area around the working table (Fig. 1).

Video recording of each milling procedure was made from the two angles of the cranial corners of the table and from the caudal edge of the table. Additionally, a tilting recording system with a cell phone in slow motion mode was used for recording zenital angles.

To collect the distribution of the drops around the corpse, semi-absorbent papers were placed with a distribution according to Fig. 2.

These semi-absorbent papers were also placed on the corpse at different heights of 20,40,50 and $55 \mathrm{~cm}$ to check vertical dispersion (Fig. 2).

The macroscopic distribution of the dyed droplets was studied by photographic analysis. The collected deposit material was analysed with the microscope (Leitz DM RB models), and its size through the image $\mathrm{J}$ v. 1.53a application, using the following magnification objectives 1, 6, 5, 10, 20, 40 and 63. Each paper was divided into eight fragments of equal size, to facilitate viewing under the microscope.

\section{Results}

The dispersion of the particles, both droplets and aerosols, was a function of their size (Fig. 3). The visualization of the coloured droplets in the horizontal plane at a magnification of 1.5 was detected at $150 \mathrm{~cm}$ from the focus of emission of milling particles.

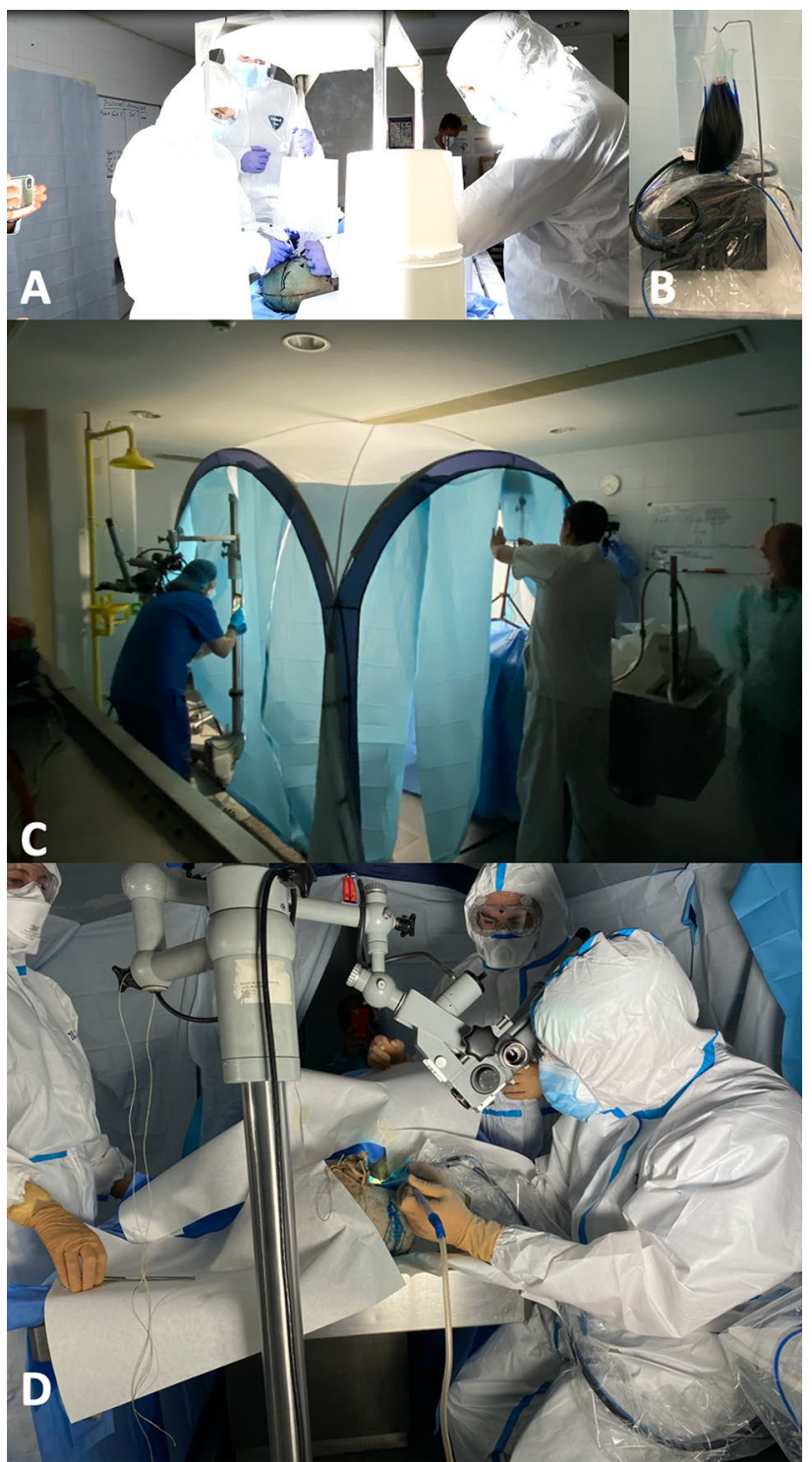

Fig. 1 Images during the study. A Positioning of a vertical panel during dissection. B Bag showing the methylene blue solution used for irrigation during drilling. C, D Layout of the tent and situation of the technical and assistance team. Health personnel wears full protective equipment

The size of the droplets obtained on the different papers, is shown in Table 1.

\section{Discussion}

SARS Cov-2 is the cause of an acute respiratory syndrome and responsible for more than 20 million cases and more than $2,000,000$ deaths worldwide so far $[9,10]$. This pandemic is unprecedented as a health crisis and has required that both clinicians and laboratory workers had had to adapt to new information, innovating and improving their usual 
Fig. 2 Horizontal (A) and vertical (B) distribution of the semi-absorbent papers with respect to the dissection table. The horizontal panels were placed on the floor coinciding with the limits of the surgical table, leaving no space without material collection. These vertical panels were placed 20, 40, 50 and $55 \mathrm{~cm}$ above the head of the corpse

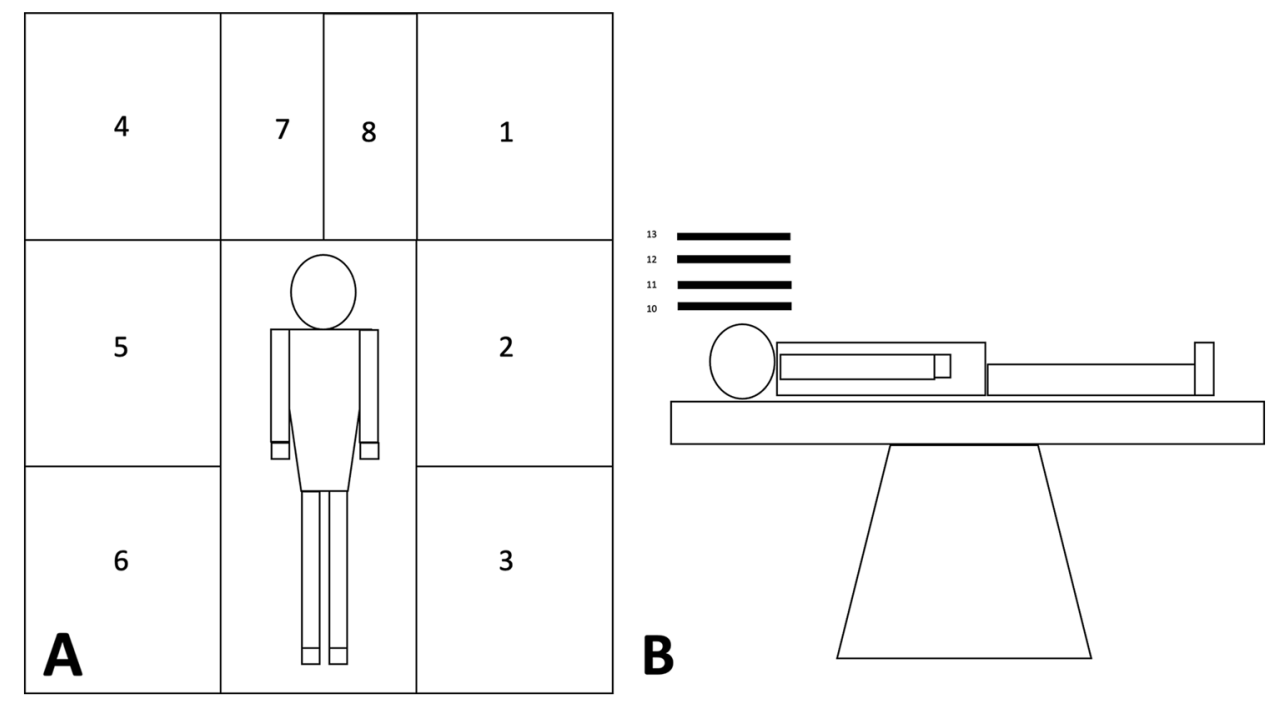

Table 1 Maximum and minimum droplet size collected according to their location

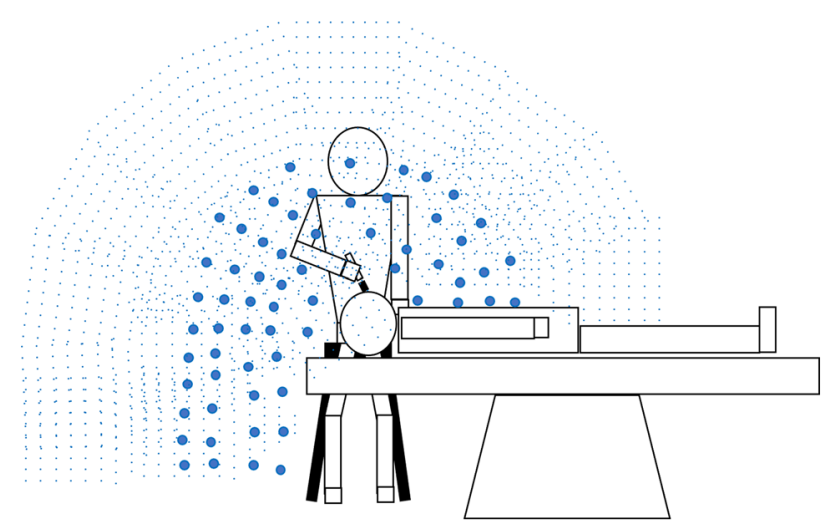

Fig. 3 Dispersion of droplets and aerosols during temporal bone dissection with $80,000 \mathrm{rpm}$ motor

practices to achieve the same efficiency in new protective situations [3].

It is generally considered that respiratory viral infections can be spread by direct contact with an infected person or surfaces that may be contaminated by the virus, on which the virus may remain stable, as well as by aerosols. The latter can be classified into droplets or fine droplets. While a droplet comprises those particles that are capable of precipitating rapidly to the ground at a maximum distance of $1 \mathrm{~m}$, fine droplets are much smaller and can remain suspended in the air and travel greater distances [3,11]. According to the World Health Organization, the cut-off point of $5 \mu \mathrm{m}$ establishes the difference between fine droplets, smaller than five, and droplets, larger than $5 \mu \mathrm{m}$. After conducting our experimental study, this classification proves to be insufficient. First, we have been able to locate particles of up to $8 \mu \mathrm{m}$ at a distance of more than $1 \mathrm{~m}$, exceeding the above-mentioned limits. That is, larger particles can reach further. Second, we have only demonstrated the presence of particles of $2 \mu \mathrm{m}$

\begin{tabular}{lll}
\hline $\begin{array}{l}\text { No. paper on the table } \\
\text { or floor }\end{array}$ & Droplet minimum size $(\mu \mathrm{m})$ & $\begin{array}{l}\text { Droplet } \\
\text { maximum } \\
\text { size }(\mathrm{cm})\end{array}$ \\
\hline 1 & 2 & 1.9 \\
2 & 2 & 1.8 \\
3 & 2 & 1 \\
4 & 8 & 2.4 \\
5 & 8.94 & 2.4 \\
6 & 7.20 & 2.6 \\
7 & 2 & 2.3 \\
8 & 2 & 0.8 \\
No. of paper in height & & \\
10 & 2 & 0.45 \\
11 & 4 & 0.28 \\
12 & 6 & 0.20 \\
13 & 6 & 0.20 \\
\hline
\end{tabular}

Panel number 9, positioned vertically at head level, was dismissed due to deterioration during the study

(Fig. 4), assuming that the smaller ones remain floating at least longer than $3 \mathrm{~h}$, the sample collection time considered (surgical approach and one more hour of rest). We consider that there could be a bias in this collection time, the influence that methylene blue could have on the weight of the droplets, the use of high-speed drills that propel particles with greater force, and in the measurement of the droplets as they could look bigger when they are absorbed by the soaker. We have to point out that, during a trans-labyrinthine approach, the particles would not precipitate, since a $1-\mu \mathrm{m}$ particle can be suspended for up to $12 \mathrm{~h}$ with a viability, in the case of SARS CoV-2, above $3 \mathrm{~h}$ in the aerosol form and up to $84 \mathrm{~h}$ in droplets $[4,6,7]$. This is not the case in middle 
Fig. 4 Microscopic images of droplets under $5 \mu \mathrm{m}$
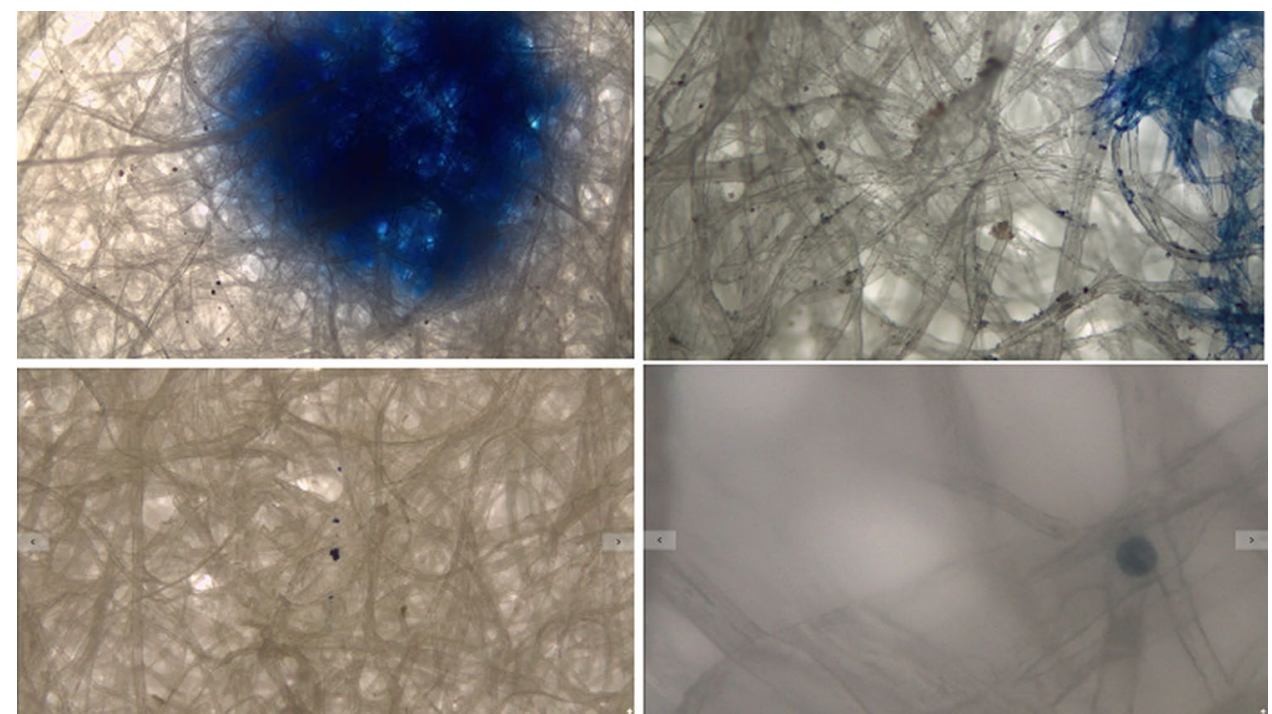

ear surgery, which is of shorter duration, and we can assume that the collection time reproduces the common situation of the total surgical time. Therefore, as proposed by Milton [5], we prefer to divide the aerosols hereby studied into respirable $(<5 \mu \mathrm{m}$ ), thoracic (up to $10-15 \mu \mathrm{m}$ ) or inhalable (up to $100-200 \mu \mathrm{m}$ ) since, for a respiratory viral process with the capacity to infect the entire surface of the respiratory tree and conjunctival mucosa, all these fractions of exposed particles are contagious [6].

For the normal population, frequent hand washing and physical distance may be sufficient to limit exposure to droplets and sprays of substance, however, it will be of little use in blocking aerosols between 20 and $30 \mu \mathrm{m}$ that can travel long distances. For these situations, the use of surgical masks has been proposed $[4,11]$. The protective capacity of the different masks has been established according to the quantity of $0.06-\mu \mathrm{m}$ particles that they are capable of blocking with an air flow of $85 \mathrm{~L}$ per minute, similar to that of a tachypnoea, expressed as a percentage. The effectiveness of surgical masks varies between 4 and $90 \%$, so surgical masks are not effective for aerosol protection. However, they can be useful in blocking saliva droplets in both directions [12]. Filtering facepiece masks 2 and 3 (FFP2 and FFP3) filter, respectively, 94 and 99\%, respectively, provided they are well adjusted to the facial surface. If this premise is not met, their failure rate can reach $60 \%$ [6]. Even with a $99 \%$ filtration, an infected person could release particles smaller than $0.06 \mu \mathrm{m}$, for which we do not know the degree of protection [13].

According to the AAO-HNS we can differentiate between two groups of patients at risk. First, those positive for SARS CoV-2, patients with diagnosed COVID-19 or under investigation with their PCR pending and emerging procedures with an unknown infection status. Second, an intermediate risk group consisting of patients who have been tested SARS
CoV-2 negative and asymptomatic patients who have not yet been tested [14].

As clinical activities on patients with coronavirus may overlap with routine clinical practice, the risk of encountering at-risk patients is possibly increasing. In this context, the study of particle dispersion during the described otologic procedures becomes important $[7,15]$ since healthcare personnel might get exposed to the aerosols typically produced during these surgical procedures. According to the literature [7] and confirmed in our study additional airbone particles will be produced by surgical drilling of bone with high speed motors of up to 80,000 rpm under continuous saline irrigation in a haemorrhagic surgical field, increasing the amount of aerosols by atomization that will persist suspended in the air for hours, particularly, those smaller than $2 \mu \mathrm{m}$.

In this situation, the use of N95 or FFP2 masks, Powered Air Purifying Respirator (PAPR), eye protection (goggles or non-ventilated eye protection), waterproof gown and gloves is recommended in the operating theatre [12]. However, the risk does not end in the operating rooms as they are equipped with positive pressure systems that minimize the entry of air but maximise the exit of contaminated air out of the operating room despite the fact that the positive pressure system is interrupted every time a door is opened. Although we have not measured the presence of particles outside of our autopsy room or beyond the scattering radius considered in material and methods, we have not demonstrated the uptake of particles smaller than $2 \mu \mathrm{m}$ for $3 \mathrm{~h}$. Therefore, it could be assumed that these smaller particles remain suspended in the air with capacity to disperse beyond these limits. However further studies should we performed measuring dispersion further, for longer time and reproducing the laminar flow of a theatre.

Subsequently, the guidelines on the use of protective equipment in operating theatres should especially be revised 
for aerosol-generating surgeries. Thus, the use of N95, FFP2 or FFP3 protective masks should be considered (including personnel and patients), advising against the use of surgical masks alone. PAPRs have been shown to offer the best protection in these circumstances, their use is usually recommended to people involved in the intervention, which could leave some personnel not fully protected. Also, handling of the microscopes wearing these suits is neither easy nor comfortable. It would be very useful to develop hood systems that would prevent the dispersion of aerosols during these surgical procedures generating aerosols or at least during the phase of generation of the greatest amounts of aerosols, limiting their dispersion and improving the safety of healthcare personnel.

The authors state that there are no conflicts of interest and that there has been no external financing.

Acknowledgements The authors would like to thank Professor Manuel Bernal-Sprekelsen for his help and guidance during the completion of this work.

Author contributions MGS and ASS designed the study. WASB, ALS, RMR and MGS performed the dissection and AFGM, AS and JAGG prepared the study field and performed the recording, photography and measurement of the results. APPB, JGH and MGS performed the bibliographic research. MCI, ASS and MGS wrote the manuscript.

Funding The authors report no conflicts of interest.

Availability of data and material Data available at the Department of Legal Medicine, Hospital Clínico San Carlos.

\section{Declarations}

Conflict of interests The authors report no conflicts of interest.

Ethics approval and consent to participate The research work received a favourable opinion as a "Biomedical Research Project with code C.I. 20/437-E" on May 21, 2020 by the Clinical Trials Ethics Committee of the Hospital Clínico San Carlos de Madrid (Spain)".

Consent for publication Not applicable.

\section{References}

1. Saadi RA, Bann D V., Patel VA, Goldenberg D, May J, Isildak H. A Commentary on Safety Precautions for Otologic Surgery during the COVID-19 Pandemic. Otolaryngol - Head Neck Surg (United States). 2020;1-3.

2. Morawska L (2006) Droplet fate in indoor environments, or can we prevent the spread of infection? Indoor Air 16(5):335-347

3. Morawska L, Cao J (2020) Airborne transmission of SARSCoV-2: the world should face the reality. Environ Int 139(April):105730. https://doi.org/10.1016/j.envint.2020.105730

4. Jayaweera M, Perera H, Gunawardana B, Manatunge J (2020) Transmission of COVID-19 virus by droplets and aerosols: a critical review on the unresolved dichotomy. Environ Res 188(January):1-18

5. Milton DK (2020) A Rosetta stone for understanding infectious drops and aerosols. J Pediatr Infect Dis Soc 9(4):413-415

6. Fennelly KP (2020) Particle sizes of infectious aerosols: implications for infection control. Lancet Respir Med 8:914-924

7. Mick P, Murphy R (2020) Aerosol-generating otolaryngology procedures and the need for enhanced PPE during the COVID19 pandemic: a literature review. J Otolaryngol Head Neck Surg 49(1):1-10

8. Bałazy A, Toivola M, Adhikari A, Sivasubramani SK, Reponen T, Grinshpun SA (2006) Do N95 respirators provide 95\% protection level against airborne viruses, and how adequate are surgical masks? Am J Infect Control 34(2):51-57

9. Worlometer. COVID-19 coronavirus pandemic. https://www. worldometers.info/coronavirus/. Accessed 7 Mar 2021

10. Control EC for DP and. COVID-19 situation update worldwide. https://www.ecdc.europa.eu/en/geographical-distribution-2019ncov-cases. Accessed 7 Mar 2021

11. WHO (2020b) WHO announces COVID-19 outbreak a pandemic. http://www.euro.who.int/en/health-topics/health-emergencies/ coronavirus-covid-19/news/news/2020/3/who-announces-covid19-outbreak-a-pandemic. World Health Organisation. Accessed Feb 2021

12. Howard BE (2020) High-risk aerosol-generating procedures in COVID-19: respiratory protective equipment considerations. Otolaryngol Head Neck Surg 163(1):98-103

13. Gralton J, Tovey E, McLaws ML, Rawlinson WD (2011) The role of particle size in aerosolised pathogen transmission: a review. $\mathrm{J}$ Infect 62(1):1-13

14. Kozin ED, Remenschneider AK, Blevins NH, Jan TA, Quesnel AM, Chari DA et al (2020) American Neurotology Society, American Otological Society, and American Academy of Otolaryngology-Head and Neck Foundation Guide to enhance otologic and neurotologic care during the COVID-19 pandemic. Otol Neurotol 41(9):1163-1174

15. de Simone B, Chouillard E, Di Saverio S, Pagani L, Sartelli M, Biffl WL et al (2020) Emergency surgery during the COVID-19 pandemic: what you need to know for practice. Ann R Coll Surg Engl 102(5):323-332

Publisher's Note Springer Nature remains neutral with regard to jurisdictional claims in published maps and institutional affiliations. 


\section{Authors and Affiliations}

\section{Manuel Gomez Serrano ${ }^{1}$ (1) - Andres Santiago-Saez ${ }^{2} \cdot$ Rosa Moreno Rodriguez $^{3} \cdot$ Andrea Lopez Salcedo $^{1}$. Adriana Poch Perez-Botija ${ }^{1}$. William Aragones Sanzen-Baker ${ }^{1}$. Angel F. García Martín ${ }^{4}$. Juan Antonio García García ${ }^{5}$. Maria Cruz Iglesias Moreno ${ }^{1}$}

Andres Santiago-Saez

Ansantia@ucm.es

Rosa Moreno Rodriguez rmorenor@madrid.salud.org

Andrea Lopez Salcedo mals44@gmail.com

Adriana Poch Perez-Botija adri_poch@hotmail.com

William Aragones Sanzen-Baker william.aragones@hotmail.com

Angel F. García Martín garciamaf57@gmail.com

Juan Antonio García García jagarciag@salud.madrid.org
Maria Cruz Iglesias Moreno mcruziglesias@hotmail.com

1 ENT Department, Servicio de Otorrinolaringología, Hospital Clínico San Carlos, Universidad Complutense, Calle Profesor Martin Lagos s/n. $5^{\text {a }}$ Planta, Madrid, Spain

2 Head of Legal Medicine Department, Professor School of Medicine, Hospital Clínico San Carlos, Universidad Complutense, Madrid, Spain

3 Hospital Clínico San Carlos, Madrid, Spain

4 Madrid Salud, Department of Legal Medicine, Psychiatry and Pathology, School of Medicine, Institute of Public Health, Universidad Complutense, Madrid, Spain

5 Anaesthetic Department, Hospital Universitario Infanta Cristina, Madrid, Spain 